\title{
Reducing SNF Readmissions: At What Cost?
}

\author{
Robert E. Burke, MD, MS ${ }^{1 *}$, S. Ryan Greysen, MD, MHS, MA²
}

'Denver VA Medical Center-Research and Hospital Sections, University of Colorado Medical Center-Medicine, Denver, Colorado; ${ }^{2}$ Hospital of the University of Pennsylvania, Philadelphia, Pennsylvania.

rast he landscape of postacute care in skilled nursing facilities (SNFs) in the United States is evolving. As the population ages, a growing number of elderly persons are being discharged to SNFs at an enormous cost and with clear evidence of disappointing outcomes. The reaction to these trends includes payment reforms that "bundle" hospital and postacute care, act as incentives to discourage SNFs, or penalize SNFs for undesired patient outcomes. Hospitalists are expected to increasingly feel the effect of these reforms.

Thus, hospitals are demonstrating renewed interest in reducing readmissions from SNFs. In this issue of Journal of Hospital Medicine, Rosen and colleagues present the results of the Enhanced Care Program (ECP), a multicomponent intervention consisting of 9 nurse practitioners (NPs), a pharmacist, a pharmacy technician, a nurse educator, a program administrator, and a medical director. ${ }^{2}$ These providers are deployed to 8 SNFs around a large teaching hospital, providing direct clinical care as well as $24 / 7$ call availability for enrolled patients, robust medication reconciliation, and monthly education for SNF nursing staff. A unique aspect of this model was that individual attending physicians in the associated SNFs could decide whether to enroll their patients in the model; patients not enrolled represented a contemporaneous control cohort. The authors found a nearly $30 \%$ reduction in the odds of 30 -day readmission (OR 0.71 [0.60-0.85] after adjustment), which was robust to multiple sensitivity analyses, including a propensity-matched cohort comparison. The authors should be commended for working to mitigate these potential confounders, thereby strengthening their conclusions. Such a large reduction in readmissions reflects their high underlying prevalence (23\% in the nonintervention cohort).

This report closely follows the evaluation of a similar program at the Cleveland Clinic called Connected Care Model (CCM), in which 4 physicians and 5 NPs or physician assistants provided care, including 24/7 call availability, in 7 associated SNFs. ${ }^{3}$ In a retrospective pre-post analysis comparing the 30 day readmission rates of these SNFs with those of others in the network, similar reductions in readmissions were observed. ECP and CCM represent important extensions of a much larger body of evidence, from the Evercare model ${ }^{4}$ to the Initiative

\footnotetext{
*Address for correspondence: Robert Burke, MD, MS, Denver VA Medical Center, 1055 Clermont Street, Denver, CO 80220; Telephone: 303-393-8020; Fax: 303-393-5199; E-mail: Robert.Burke5@va.gov
}

Received: February 1, 2018; Accepted: February 7, 2018

2018 Society of Hospital Medicine DOI 10.12788/jhm.2970 to Reduce Avoidable Hospitalizations demonstration project, which suggests that adding NPs to nursing homes reduces hospitalizations. ${ }^{5}$

However, several factors have to be considered before disseminating ECP or CCM. First, other promising "proof of concept" quality improvement studies were not efficacious when rigorously tested in nursing homes. ${ }^{6}$ Second, these programs are representative of large academic medical centers, which may establish different relationships with different SNFs compared with smaller or less well-resourced hospitals. As the Initiative to Reduce Hospitalizations demonstrated, even a fundamentally similar intervention can have extremely different results depending on the nursing homes involved, ${ }^{5}$ and the science behind establishing effective hospital-SNF partnerships is still in its infancy. ${ }^{7}$ Third, both studies have significant methodological limitations, including most importantly that they are conducted within SNFs selected to be part of their hospitals' network.

These significant early efforts also present an opportunity to reconsider the underlying assumption of these models: that adding more supervisory clinicians to SNFs is the right approach to reduce hospitalizations. Although adding resources is an attractive "plug and play" solution for many problems in healthcare delivery, placing only 1 NP in each of the 15,583 certified nursing facilities in the United States would employ fully $10 \%$ of the entire NP workforce. Amid rising concerns about costs related to our aging population, these interventions face substantial headwinds toward becoming the standard of care without demonstrating cost effectiveness. Furthermore, many SNF directors might suggest that hospitals and hospitalists working with them to address fundamental (but much more intransigent) problems in SNFs, such as high staff turnover, low concentration of highly skilled staff (RNs and MDs), regulatory burden, and hospitals using SNFs like stepdown units, could represent a generalizable and sustainable solution.

We realize that this argument is tricky for hospitalists because its underlying logic (care has become too complex, patients are too sick, and dedicated personnel are needed) also played a major role in establishing our existence. One possibility is that like hospitalists, NPs and a growing cadre of "SNFists" will become major drivers of quality improvement, education, and leadership locally at these facilities, thereby leading to sustainable change. ${ }^{8}$ Similarly, current conditions may drive recognition that a specific set of skills is required to function effectively in the SNF environment, ${ }^{9}$ just as we believe hospitalists need unique skills to excel in today's hospital environment.

Studies such as that of Rosen et al. are valuable for JHM because they prompt us to recognize that we as hospitalists have 
much to share and learn from nursing homes and the dedicated practitioners who work there. In fact, we argue that few places in the healthcare system are more in need of innovation than hospital-nursing home relationships, and hospitalists do not just have a vested clinical interest; in many ways, we see a mirror of our own development as a "specialty." We encourage hospitals and hospitalists to take up this challenge on behalf of some of the most vulnerable patients in our system during crit-

\section{References}

1. Burke RE, Cumbler E, Coleman EA, Levy C. Post-acute care reform: Implications and opportunities for hospitalists. J Hosp Med. 2017;12(1):46-51. 10.3810/hp.2012.02.958

2. Rosen BT, Halbert RJ, Hart K, Diniz MA, Isonaka S, Black JT. The enhanced care program: Impact of a care transitions program on 30-day hospital readmissions for patients discharged from an acute care facility to skilled nursing facilities. J Hosp Med. 2018;13(4):229-235.

3. Rothberg MB. Impact of a connected care model on 30-day readmission rates from skilled nursing facilities. J Hosp Med. 2017;12(4):238-244. 10.12788/ jhm.2710.

4. Kane RL, Keckhafer G, Flood S, Bershadsky B, Siadaty MS. The effect of Evercare on hospital use. J Am Geriatr Soc. 2003;51(10):1427-1434. 10.1046/j.15325415.2003.51461.x.

5. Ingber MJ, Feng Z, Khatutsky G, et al. Initiative to reduce avoidable hospital- ical times in their care trajectory. As the Commission for LongTerm Care (www.ltccommission.org) wrote in its final report to Congress: "The need is great. The time to act is now."

Disclosures: Dr. Burke is supported by a VA Health Services Research and Development (HSR\&D) Career Development Award. All opinions are those of the authors and do not necessarily represent those of the Department of Veterans Affairs. Dr. Greysen has nothing to disclose.

izations among nursing facility residents shows promising results. Health Aff Proj Hope. 2017;36(3):441-450. 10.1377/hlthaff.2016.1310.

6. Kane RL, Huckfeldt $\mathrm{P}$, Tappen $\mathrm{R}$, et al. Effects of an intervention to reduce hospitalizations from nursing homes: A randomized implementation trial of the INTERACT Program. JAMA Intern Med. 2017;177(9):1257-1264. 10.1001/ jamainternmed.2017.2657.

7. Lage DE, Rusinak D, Carr D, Grabowski DC, Ackerly DC. Creating a network of high-quality skilled nursing facilities: preliminary data on the postacute care quality improvement experiences of an accountable care organization. J Am Geriatr Soc. 2015;63(4):804-808. 10.1111/jgs.13351.

8. Ryskina KL, Polsky D, Werner RM. Physicians and advanced practitioners specializing in nursing home care. JAMA. 2017;318(20):2040-2042. 10.1001/jama.2017.13378.

9. Gillespie SM, Levy CR, Katz PR. What exactly is an "SNF-ist?" JAMA Intern Med. 2018;178(1):153-154. 10.1001/jamainternmed.2017.7212. 